\title{
Corela
}

Cognition, représentation, langage

HS-28 | 2019

Les affordances langagières : textualité numérique, matérialité discursive

\section{Nature et domotique du langage : Une approche des interactions naturelles homme-machine en environnement pervasif}

\section{François Perea}

\section{OpenEdition}

\section{Journals}

Édition électronique

URL : http://journals.openedition.org/corela/8498

DOI : $10.4000 /$ corela.8498

ISSN : $1638-573 \mathrm{X}$

Éditeur

Cercle linguistique du Centre et de l'Ouest - CerLICO

Référence électronique

François Perea, « Nature et domotique du langage : Une approche des interactions naturelles hommemachine en environnement pervasif », Corela [En ligne], HS-28 | 2019, mis en ligne le 11 septembre 2019, consulté le 13 septembre 2019. URL : http://journals.openedition.org/corela/8498 ; DOI : 10.4000/corela.8498

Ce document a été généré automatiquement le 13 septembre 2019.

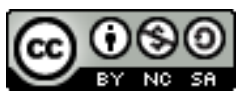

Corela - cognition, représentation, langage est mis à disposition selon les termes de la licence Creative Commons Attribution - Pas d'Utilisation Commerciale - Partage dans les Mêmes Conditions 4.0 International. 


\title{
Nature et domotique du langage : Une approche des interactions naturelles homme-machine en environnement pervasif
}

\author{
François Perea
}

\section{Nature et mécanique des interactions naturelles homme-machine domestiques}

L'évolution industrielle nous destine à vivre dans des environnements technologiques de plus en plus pervasifs ou ubiquitaires (Weiser, 1991), c'est-à-dire caractérisés par une dissémination informatique totale dans la multitude croissante d'appareils et d'objets connectés nous entourant, aux systèmes attentifs et intelligents capables de détecter et «interpréter" de manière autonome les actions des objets ou des personnes pour concevoir une nouvelle écologie d'évolution humaine. Il s'agit, pour Rafi Haladjian :

\begin{abstract}
de créer un environnement sans centre de gravité, dans lequel l'Homme n'a plus besoin d'aller au puits pour chercher (ou envoyer) de l'information. L'intelligence n'y est plus l'apanage de certains équipements dédiés, trônant au milieu d'un monde physique globalement stupide. Elle est potentiellement disséminée dans tous les éléments qui entourent l'Utilisateur (2003, p. 10).
\end{abstract}

Plus qu'une évolution technologique marquée par la miniaturisation et la multiplication des processus et artefacts informatiques, la connexion d'objets capables de traitement sémantique et d'interaction informationnelle (on dit même "cognitive») avec les humains, c'est donc une véritable révolution informatique humaniste qui est alors promise avec l'Intelligence ambiante (le terme est repris à Zelkha et al., 1998) : 
La finalité de l'«intelligence ambiante » est l'amélioration, voire l'augmentation $\mathrm{du}$ monde réel pour offrir un tout adapté en toute circonstance à l'Homme. Autrement dit, il s'agit de créer des services et des dispositifs intelligents capables de répondre à des besoins individuels, collectifs et sociétaux. Contrairement à l'informatique conventionnelle, il ne s'agit plus seulement de développer des artefacts technologiques qui améliorent l'efficacité de la personne sur son lieu de travail, mais d'améliorer le bien-être de l'individu et de la société, et de là, soutenir la sauvegarde de notre planète. [...] Cette ambition confère aux Technologies de l'Information et de la Communication un nouveau statut, celui d'être au service de l'Humanité (Coutaz et Crowley, 2008, p. 3)

Ce travail propose d'explorer quelques-uns des comportements langagiers humains dans cette tension en direction de l'intelligence ambiante, notamment dans le cadre des interactions naturelles homme-machine (désormais INHM), en tant qu'ils témoignent d'une évolution des conceptions du langage et de l'humanité.

4 Après avoir posé le cadre d'une réflexion envisageant certaines incidences des évolutions technologiques ubiquitaires sur les conceptions du langage et le cadre théorique (1. Technologies linguistiques), puis présenté les lignes d'un questionnement sur les dialogues dits "naturels» entre l'homme et la machine (2. Approche des INHM), il s'attache à présenter des éléments d'analyse de phénomènes caractéristiques dans ces interactions entre nature et artifice (3. Technologie et naturalité des échanges) à travers l'exemple du dispositif Google Home, avant de conclure sur la co-détermination de l'homme et de la machine, observable dans/par les comportements langagiers.

\section{Technologies linguistiques}

Pour comprendre la place essentielle qui est accordée aux technologies contemporaines de médiation symboliques, il est nécessaire de prendre en compte leur lien historique et structural aux humains.

\subsection{Genèse, technique et pensée}

6 Il est impossible de réduire les évolutions technologiques à des éléments extérieurs à la nature humaine, à des accessoires indépendants. La perspective de Katherine Hayles plaide au contraire en faveur « d'une autre manière d'avancer qui consiste à recourir au concept de technogenèse, qui explore l'idée d'une coévolution des humains et de la technique » (2016, p. 51). Dès lors, il n'est plus possible de considérer la technique comme un produit de l'homme sans considérer en retour que l'homme est un produit de la technique, comme le souligne Bernard Stiegler :

si l'on dit souvent que l'homme a inventé la technique, il serait peut-être plus exact ou en tout cas tout aussi légitime de dire que c'est la technique, nouveau stade de l'histoire de la vie, qui a inventé l'homme (B. Stiegler, 1998, p. 190).

Le rapport humanité / technique est ainsi co-constitutif et connait des incidences cognitives car la technologie, inscrite dans son environnement, participe ainsi à la pensée humaine. C'est notamment ce que soulignent les travaux que James Jérôme Gibson (1977) 
développe dans une approche écologique de la perception, où l'environnement (milieu et substances) afforde de possibles interactions dans un processus liant perception et action :

Je suggère que ce que nous percevons quand nous regardons des objets, sont leurs affordances, non leurs qualités (Gibson, 1979/1986, p. 134, je traduis).

8 La proposition gibsonienne et les liens opérés par la suite avec d'autres approches de la distribution de la cognition (notamment et notablement chez Hutchins), ont permis de comprendre que les processus de pensée ne constituent pas le résultat d'opérations internes au sujet, mais qu'ils apparaissent dans l'interaction du sujet avec son environnement social, matériel... Donald Norman (1989), développera pour sa part le concept d'affordance dans une approche ergonomique pour désigner les potentialités d'action qui sont perceptibles par l'utilisateur d'un programme.

9 C'est dire que la transformation ubiquitaire de notre environnement à l'intelligence ambiante n'est pas sans incidence sur l'humain et sa nature. Les modifications que ces technologies opèrent sur les manières de penser et d'agir, voire sur la définition même de l'humanité, sont accessibles et concrètement observables sur le plan langagier.

\subsection{Hier et aujourd'hui}

10 Certaines technologies reposent sur un travail linguistique. Ainsi, des traitements numériques des textes, de la traduction automatique ou encore des interactions vocales homme-machine qui travaillent la matérialité du langage pour en permettre la saisie informatique.

11 Alors, en même temps que se déploient des solutions pour rendre le langage « traitable », se dessinent des conceptions linguistiques qui affectent les sujets parlants dans leurs représentations et leurs usages langagiers.

12 Sylvain Auroux (1994) a montré comme les «révolutions» technolinguistiques se succédaient depuis l'invention de l'écriture qui marque le début de l'histoire humaine.

13 La scripturalisation est la première de ces révolutions. Elle débute par l'apparition de l'écriture il y a plus de 5300 ans en Mésopotamie et prend une ampleur remarquable avec la généralisation de l'imprimerie. Avec l'écriture apparait alors la «raison graphique » marquée par des conceptions nouvelles permises par un nouveau rapport au temps (la fixation de la parole jusqu'alors éphémère) et à l'espace (dans la bidimensionalité de l'espace planaire). L'écriture conduit au développement de compétences cognitives qui vont entraîner un changement de paradigme dans la pensée humaine. Elle est marquée par la nécessité d'une standardisation qui va aller croissant (conduisant à de nouvelles révolutions) et une transformation des comportements langagiers comme par exemple à l'obligation d'expliciter les paramètres de l'énonciation en raison de l'absence des interlocuteurs.

14 Parce qu'elle procède d'une objectivation du langage dont il faut sélectionner certaines propriétés matérialisables (sonores et sémantiques), l'écriture conduit à sa technologisation par un ensemble de représentations et de techniques linguistiques que Sylvain Auroux nomme grammatisation. Le processus conduit à « décrire et à outiller la langue sur la base de deux technologies, qui sont encore aujourd'hui les piliers de notre savoir métalinguistique : la grammaire et le dictionnaire» (1994, p. 109). Ces piliers ne 
préexistent pas (dans une sorte d'état naturel linguistique) à l'écriture: ils sont des constructions conséquentielles. Pour le linguiste, cette deuxième révolution est capitale :

[il s'agit] d'une révolution technologique aussi importante pour l'histoire de l'humanité que la révolution agraire du néolithique [qui a] profondément changé l'écologie de la communication humaine et a donné à l'Occident des moyens de connaissance et de domination sur les autres cultures de la planète » (p. 109).

Les conséquences de la grammatisation sont à la fois cognitives (le développement de la « raison graphique ») et sociales : pendant treize siècles, ses processus ont organisé les sociétés via des transferts de technologie d'une langue à l'autre, mais aussi des transferts culturels plus vastes.

Les deux étapes précèdent la troisième révolution apparue vers le milieu du XXème : l' automatisation de l'écriture (et des sciences du langage : sur ce point voir J. Léon, 2015) qui poursuit le travail de formalisation et d'externalisation du langage humain. Sylvain Auroux est ici rejoint par Bernard Stiegler pour lequel :

le numérique est le stade le plus avancé d'un processus de grammatisation [...] l'écriture numérique est opto-électroniquement enregistrée et véhiculée, elle est partiellement automatisée, elle fait appel à des ressources algorithmiques, c'est-à-dire computationnelles, dont les traitements sont effectués en réseau et transmissibles par ces mêmes réseaux, ce qui transforme très rapidement et radicalement la plupart des aspects caractéristiques de l'existence humaine en général, y compris dans ce qu'elle a de plus intime (2013, en ligne).

17 Le déploiement des codes informatiques participe des dernières formes de grammatisation. Katherine Hayles (2016) voit dans le développement des codes télégraphiques l'une de ces sources : «L'interaction entre code [télégraphique] et langage montre le passage d'une vision du code centré sur les humains à une vision centrée sur la machine, anticipant ainsi l'élaboration de codes machine à part entière avec l'ordinateur numérique » (p. 213). Parmi les conséquences de ce passage, l'auteure souligne l'absence d'intervention humaine (les machines communiquant entre elles) et une perception du langage déclinés en couches :

Une révolution s'est produite dans la pratique de la langue, avec des conséquences théoriques importantes, quand on est passé de l'idée du codage/décodage comme un déplacement à travers la page imprimée à celle d'un déplacement vers le haut ou vers le bas entre différentes couches de code et de langue. [...] Positionné au niveau de la couche inférieure, le code binaire est devenu le code universel dans lequel tous les autres langages, ainsi que des images et de sons, pouvaient être traduits. Contrairement aux codes télégraphiques, qui positionnaient les langues nationales comme les langages "naturels » auquel on faisait correspondre des groupes de lettres de chiffres " artificiels ", les surfaces linguistiques peuvent être considérées, dans le contexte de l'informatique, comme des épiphénomènes engendrés par le code sous-jacent (2016, p. 263).

18 L'une des incidences les moins «visibles » de cette grammatisation évolutive a trait au traitement automatisé de l'oral. Il n'est pas nouveau: l'écrit repose pour partie une transcription de l'oral, et le traitement des reproductions vocales et sonores a, au cours du XXe siècle en particulier, été largement déployé dans des dispositifs technologiques. 


\section{consolidée permettait une normalisation poussée. Leur traitement est désormais incontournable dans les environnements pervasifs où les gens parlent et dialoguent comme ils le font depuis la nuit des temps: en se coupant la parole, en référant implicitement à la situation, en usant des sous-entendus, en parlant parfois même pour ne rien dire mais pour le plaisir d'être ensemble, de faire de "l'épouillage verbal» (Dunbar, 1998), etc. Les interactions homme-machine, pour poursuivre leur évolution, doivent donc être capables de gérer ces formes dites "naturelles» et d'inclure les échanges «spontanés » avec les machines parlantes dans des procédés conduisant à leur formatage. \\ 2. Approche des Interactions Naturelles Homme- Machine (INHM)}

interessants, peut-être parce que moins formatables que l'écrit dont la grammatisation

Du côté des sciences du langage, les avancées ont été remarquables dans le champ de la linguistique informatique et notamment du traitement automatique de la langue et de la parole (sur ce point, voir par exemple les numéros 141 et 142 de la revue L'Information grammaticale) qui a permis un travail sur les modélisations linguistiques. Au-delà de ces technologies de la langue, les technologies relationnelles multicodiques (interactionnelles, discursives...) ont également été développées car dans ce nouveau monde, les humains ont à se mouvoir sans cesse dans des flux numériques générant et déterminant les interactions avec leurs congénères et les objets.

\subsection{Une approche en sciences du langage}

23 Le présent travail s'inscrit dans le cadre d'une linguistique non dualiste et non logocentrée (TDI) dans lequel des éléments de simulacre conversationnel sont particulièrement explorés.

\subsubsection{Textes - discours - interactions}

Ce cadre de recherche TDI proposé par M.-A. Paveau (2012a), propose une approche en sciences du langage symétrique (en référence à B. Latour, 1997) considérant les rapports 
entre humains et non-humains dans un continuum, et le social dans une dimension intersubjectives et interobjectives assemblées. Dès lors...

Les objets inanimés, qui ont une âme comme chacun sait, ont aussi une bouche : ils parlent, souvent, et beaucoup. Mais on ne les écoute sans doute pas assez (Paveau, 2012a, p. 54).

Il convient donc de questionner «ce que disent les objets» (Paveau, 2012) et « les efforts que déploient les humains pour interagir avec l'artefact » (Hutchby, 2001). Pour ce faire, Marie-Anne Paveau (2012, p. 61) distingue alors :

- les outils soutenant les compétences langagières en les enregistrant (outils linguistiques tel le dictionnaire), et/ou permettant le discours (outils discursifs tel le plan d'une conférence que suit l'orateur);

- es objets supportant les discours en étant support d'inscription (discursifs graphique, tel un emballage alimentaire) ou proposant des usages et agir discursifs (non graphiques, tel le distributeur de café au travail).

Pour rendre compte des INHM, il est nécessaire de complèter la liste par la prise en compte des objets parlants interactionnels, artefacts spécifiquement dédiés à être partenaires de dialogue avec un humain de manière linguistique (dans les cas qui nous intéressent ici) ou pas.

En réalité, un continuum existe entre les objets discursifs et les objets interactionnels. Cependant, ces derniers se distinguent en n'étant pas «que» des vecteurs d'inscription: ils proposent une adaptation aux comportements langagiers humains, une forme de réaction qui peut être basique (comme le répondeur téléphonique peut router l'appelant qui a énoncé «service technique » ou "service commercial») ou complexe (comme c'est le cas avec des agents conversationnels domotiques par exemple). Ces dispositifs s'inscrivent donc dans le cadre d'un agir interactionnel, entre un agent humain et un agent non humain. Ils proposent des modes d'interactions diversement orientés: de la machine vers l'homme (comme avec la requête d'un GPS), de l'homme vers la machine (comme avec les requêtes vocales dans certains systèmes domotiques) ou dans les deux sens (systèmes de dialogue).

Dans ce dernier cas, les objets ne parlent plus en tant que tels, mais comme des sujets parlants identifiés et naturels. Dès lors, ce ne sont plus seulement des principes d'affordances qui leur donnent voix, mais véritablement une compétence discursive et interactionnelle, simulant le face-à-face humain, présentiel ou distanciel (Develotte et al., 2011) selon les dispositifs. Ces différents aspects se fondent particulièrement dans le cadre des environnements connectés et pervasifs.

A l'intérieur du cadre TDI, le présent travail propose des éléments d'analyse issus de l'analyse des conversations (cf. 3.2) appliqués au dispositif Google Home qui servira d'exemple d'ancrage. Il s'agit d'« une enceinte à commande vocale qui fonctionne avec l'Assistant Google » (https://madeby.google.com/home/, consulté le 04/10/2017). L'approche est double puisqu'elle s'attache :

- au dispositif IHNM, reposant sur certaines configurations des comportements des agents humains et non humains ;

- à l'objet de communication pour la firme Google qui promeut son «identité » et le couplage à l'humain sur les pages de son site, en un discours préparant les représentations qui participent à orienter les expériences des utilisateurs. 
deux corpus sont extrêmement liés, en particulier dans le contexte d'un lancement de produit où le discours publicitaire vise à influencer, si ce n'est à cadrer (au sens goffmanien), l'usage effectif et son interprétation. Ce discours promotionnel, par définition même, vise à impulser un mouvement interprétatif, à constituer ici ce que l'on pourrait appeler un horizon d'usage. Précédent l'expérience, il devient prégnant et devenir préconstruit tel que définit Michel Pêcheux :

\section{[...] le préconstruit, tel que nous l'avons redéfini renvoie simultanément à "ce que chacun connaît", c'est-à-dire aux contenus de pensée du "sujet universel » support de l'identification et à ce que chacun, dans une «situation » donnée, peut voir et entendre, sous la forme des évidences du « contexte situationnel ». (Pêcheux, 1975, dans Maldidier, 1990, p. 235).} machines. Certes, des technologies ont depuis longtemps permis les interactions humaines (d'une manière générale, les dispositifs médiatiques), certaines en révélant même la structure conversationnelle (par exemple avec les phylactères latéralisés affectant chaque position énonciative dans les SMS). Cependant, la participation conversationnelle globale, incluant la production de l'énoncé du tour, était jusqu'à il y a peu réservée au genre de la fiction anticipatrice. Désormais, l'artefact parle, répond, presque comme le ferait une personne présente dans la pièce. d'autant plus prégnant qu'il se déploie dans un environnement «transparent»: l'utilisateur n'est plus face à une machine (l'écran d'un ordinateur, d'une tablette ou d'un téléphone) mais dans son environnement domestique ordinaire, vacant à ses occupations dans lesquelles viennent s'intriquer le dispositif, sur le mode d'une présence discutante.

Cette extension du champ de l'informatisation du langage humain invite à appliquer une lecture conversationnelle de ces échanges. Adopter une telle lecture suppose de s'intéresser, plus qu'aux phénomènes de reconnaissance et de production de la chaine vocale ou au traitement de l'information, aux dimensions structurantes, structurelles et sociales des échanges entre sujets. Il s'agit, à l'instar de Robin Dunbar (1998), de considérer que les conversations visent, avant tout partage de "contenu ", à créer et signifier des réseaux de sociabilité complexes et privilégiés, en une activité proche de l'épouillage des primates. C'est donc une dimension de coordination à la fois sociale et affective (qui parle à qui et comment) et praxéologique (qui agit avec qui et comment) qui est mise en avant. Et Michel de Fornel et Jacqueline Léon d'écrire :

L'analyse de conversation considère qu'il est essentiel d'étudier l'interaction en tant que processus complexe de coordination des actions et en tant qu'accomplissement pratique. Lorsqu'ils sont en relation de co-présence, les participants à une interaction se rendent mutuellement intelligibles le sens 
de leurs actions et la compréhension qu'ils ont de ce qui se passe (2000, p. 144). importance car, au-delà d'une signification première et technique, il permet de déployer un simulacre de comportement tout en cadrant l'activité humaine et langagière. Ce naturel est ainsi, pour parler comme Sylvain Auroux, le signe d'un stade ultime de l'automatisation non plus de l'écriture mais de l'activité première et originelle du langage : l'oral conversationnel spontané sur lequel est posé un cadre contraignant et structurant,

\subsubsection{Acception de naturel}

41 Au-delà du cas de Google Home, il ne fait nul doute que ces dispositifs de connexion permanente vont aller croissant, avec le développement de l'intelligence artificielle et des environnements lourdement connectés. Dans cet écosystème, les interactions peuvent être latentes, automatiques et discrètes comme l'attentivité suppose une saisie constante et un croisement des données, ou peuvent s'établir sur la base de la volonté de l'un des agents (humain ou pas) depuis le mode de la requête jusqu'à celui de la conversation (Perea, 2016). Dans tous les cas, le caractère naturel de l'échange est mis en avant pour désigner la capacité à réaliser une communication multicodique sans effort apparent car calquée sur un comportement déjà ancré dans les praxis humaines :

\footnotetext{
J'appelle interaction "naturelle» une interaction qui se rapproche du comportement naturel. Ainsi, par exemple, le fait de prendre une tasse virtuelle dans la main, par l'intermédiaire d'un système de reconnaissance gestuel associée à un gant numérique, pour la poser sur une table est naturel. Par contre, le fait de cliquer sur un bouton pour demander à ce que la tasse soit mise sur la table n'est pas naturel (Touraine, 2003, p. 24).
}

Les procédés de naturalisation ${ }^{1}$ ont été déployés au-delà de la similarité comportementale dans les espaces connectés et déconnectés (l'exemple de la tasse de Touraine ci-dessus) pour permettre une saisie et une spécularité des comportements entre les agents humains

Corela, HS-28 | 2019 
et non humains. En particulier, la prise en compte des données posturo-gestuelles et de la reconnaissance et de la synthèse vocales ont conduit à des développements importants dans le champ des INHM. Ces développements technologiques ont plusieurs conséquences parmi lesquelles le déploiement de dispositifs qui se sont infiltrés dans notre quotidien de manière discrète mais croissante, capables de converser de manière naturelle. Ainsi, des assistants vocaux numériques (Siri pour Apple, Cortana pour Microsoft ou Google Now pour Android, pour ne reprendre que les programmes les plus répandus) dont les fabricants mettent en avant la qualité de la relation interpersonnelle dans le cadre du dialogue amical :

«Siri, devrais-je appeler mon ex?». Demandez à Siri s'il est judicieux d'appeler telle ou telle personne (par exemple, votre ex), et voyez ce qu'il vous conseille de faire... (https://www.apple.com/fr/ios/siri/)

43 A partir d'un noyau initial où naturel désigne l'utilisation du langage ordinaire (et non informatique), le sens s'est élargi pour inclure la naturalité de la relation, c'est-à-dire une prise en compte des données humaines (identitaires, sociales, affectives...) de l'interaction. Ce travail de la relation est total. Il repose fréquemment sur la mise en place de l'indentification des participants (Perea et Richard, 2017), qui sont nommés afin de permettre un ancrage voire un attachement relationnel, et intègre la gestion des émotions et des affects humains, que la machine doit pouvoir appréhender afin de créer un lien de proximité et d'intelligence, selon Clément Chastagnol et al. :

Robots and machines have to be sensitive to human emotions and social signals to be socially intelligent (2014, p. 200)

L'ensemble concourt à développer des systèmes de dialogues qui, au-delà de l'échange d'information, visent l'empathie "artificielle ", cognitive et émotionnelle selon S. Tisseron (2015), utiles à rendre évident, naturel, la présence de ces relations qui constituent une rupture anthropologique. En effet, si l'Homme s'est régulièrement adressé à d'autres entités qu'à des êtres humains au cours de son histoire - des dieux, des machines, parfois les deux ensemble - il ne s'est jamais attendu à ce qu'elles lui répondent sur le ton de la conversation ordinaire dans sa langue maternelle. Dans ce nouveau « couplage humano-machinique » (Sadin, 2013, p. 32), l'Homme gagne en accès à l'information mais perd son statut exclusif d'être doué de parole, sitôt que les machines sont capables de discuter comme le font les humains entre eux (Perea 2017), sur le ton amical, comme le souhaitait la firme Apple dans sa présentation de Siri sur son site :

Demandez à Siri de vous rendre service, en parlant normalement. Siri comprend non seulement ce que vous dites, mais aussi ce que vous voulez dire. Il va même jusqu'à vous répondre. Siri est si facile à utiliser, il en fait tant, que vous ne cesserez de trouver de nouveaux moyens de vous en servir [...] Adressez-vous à Siri comme à une personne [...] En fait, vous avez une véritable conversation avec votre iPhone [...] Non seulement Siri comprend ce que vous dites, mais il est assez intelligent pour comprendre ce que vous voulez dire. Il saura exactement ce que vous voulez dire et il le fera pour vous."

Il s'agit donc aujourd'hui de faire accepter cette mutation conduisant à rendre évident et naturel - justement - ce qui ne l'est pas et qui va, par retour, avoir une influence sur ce qui était auparavant évident et naturel. 


\subsubsection{Nature et mécanique}

\begin{abstract}
comportements humains. Mais elle reste machine et l'humain doit alors composer avec l'artefact en modifiant par moment son comportement naturel, de manière paradoxale vu l'ambition des dispositifs.
\end{abstract}

En effet, la machine (ainsi programmée par les humains ne l'oublions pas) doit emprunter à la «nature » du dialogue en proposant des dispositifs simulant le langage humain et masquant le code-machine. La conversation repose sur une relation interpersonnelle et donc, le déploiement d'un simulacre de traits de la personnalité humaine, avec des machines nommées (Perea et Richard, 2016), genrées (Perea 2017), personnalisées... comme avec l'assistant vocal de Microsoft, Cortana, développé en partenariat avec des scénaristes dont Deborah Harrison qui précise (2016) : «We are creating a fictional world [...] where Cortana exists ».

En même temps, l'humain doit «domestiquer» son langage naturel pour le rendre accessible, intelligible par la machine, par exemple en simplifiant la syntaxe, articulant de manière exagérée et travaillant à diminuer la polysémie lors de ses réponses aux interfaces orales. Il s'agit là d'une nouvelle forme de mécanisation (au sens de Auroux) de l'oral, différente de celle de l'écrit. En même temps, ses données personnelles sont prélevées pour permettre ce qui est appelé pudiquement «l'expérience personnalisée ». Dans le cas des assistants vocaux, cela consiste, outre les données liées à l'identité, aux activités professionnelles ou non, aux passe-temps... à donner des indications permettant de cartographier les relations sociales qui sont celles de l'utilisateur pour lui permettre d'énoncer: "Appelle Maman", "envoie un SMS à mon ex »... Google annonce par exemple qu'il recueille et utilise : «Ce que vous faites » (les sites visités, les recherches, la géolocalisation, les vidéos regardés...), «Ce que vous créez » (emails, contacts, photos, événements de l'agenda, fichiers du Google drive...) et « ce qui vous rend unique » (nom, sexe, adresse, date de naissance... " afin de rendre leurs «services plus rapides, plus intelligents et plus utiles pour vous» (https://privacy.google.com/your-data.html, consulté le 23/07/2018).

49 Ainsi, naturalité (simulation des échanges humains) et mécanisation (simplification et adaptation de l'humain à fin d'être saisi par la machine) sont deux tensions constitutives des INHM telles qu'elles se veulent de haut niveau, c'est à la rencontre des intelligences des sujets parlants dans la conversation.

\begin{tabular}{|c|c|c|c|c|c|c|c|c|c|c|}
\hline \multirow{2}{*}{$\begin{array}{l}\text { AGENT } \\
\text { HUMAIN }\end{array}$} & \multicolumn{3}{|c|}{ Mécanisation $\rightarrow$} & & & & \multicolumn{3}{|c|}{$<-$ Naturalité - - - - } & \multirow{2}{*}{$\begin{array}{l}\text { AGENT } \\
\text { NON } \\
\text { HUMAIN }\end{array}$} \\
\hline & $\begin{array}{l}\text { Identité } \\
\text { configurée } \\
\text { en } \\
\text { données }\end{array}$ & \& & $\begin{array}{l}\text { Adaptation } \\
\text { des } \\
\text { productions } \\
\text { langagières }\end{array}$ & 疱 & $\begin{array}{l}\text { DISPOSITIF } \\
\text { DNHM }\end{array}$ & 医 & $\begin{array}{l}\text { Simulacre } \\
\text { des échanges } \\
\text { interhumains }\end{array}$ & $\&$ & Personnification & \\
\hline
\end{tabular}

Le dispositif d'affordance est donc lié à cette double contrainte humano-mécanique et se déploie dans une double dimension technolinguistique : les événements conversationnels servent d'instructions programmatiques en même temps qu'ils remplissent une fonction 
conversationnelle. Par exemple, «Ok Google » permet de mettre en place le programme et de remplir la fonction d'ouverture de l'interaction. Le dispositif de programmation s'efface sous la couche qui emprunte et simule les interactions humaines. Si elle est gage de facilité et de fluidité (souvent mises en avant dans les discours promotionnels) elle est aussi parfois dispositif d'opacité car elle suppose une collecte permanente et non visible de données.

Ainsi des données personnelles sont collectées à travers la création d'un compte demandé lors de la mise en route ou dans les bases contenues dans les appareils connectés (contacts, préférences musicales, réseau familial...).

Toute personne se trouvant près de votre appareil Google Home peut lui demander des informations. Si vous avez autorisé Google Home à accéder à vos agendas, votre compte Gmail ou d'autres informations personnelles, n'importe qui peut demander ces informations à Google Home. Google Home collecte également des informations vous concernant lors de vos autres interactions avec les services Google [...] Vos conversations avec votre appareil sont enregistrées sur des serveurs installés dans des centres de données appartenant à Google (https://support.google.com/googlehome/ answer/7072285, consulté le 30/12/2017).

Il en va de même pour les données acoustiques car pour que le dispositif Google Home comme de nombreux autres - se mettent en route à l'énoncé « Ok Google », il faut qu'il écoute en permanence les interactions dans l'attente de son interpellation:

Google Home écoute de brefs extraits de conversation (ne dépassant pas quelques secondes) jusqu'à ce qu'il détecte le mot-clé. Tant que le mot-clé n'est pas détecté, ces extraits sont supprimés et aucune information ne sort de l'appareil. Lorsque Google Home détecte le mot-clé "Ok Google" ou lorsque vous touchez longuement le haut de votre appareil [...] Google Home enregistre les mots que vous prononcez et envoie cet enregistrement (y compris l'enregistrement de mot-clé de quelques secondes) à Google afin de vous proposer une réponse

(https://support.google.com/googlehome/answer/7072285, consulté le 30/12/2017).

Cette dimension double créé un réseau complexe d'affordances qui se déploie sur des traits saillants de la conversation.

\section{Technologie et naturalité des échanges}

J'ai pu proposer (Perea, 2016) une grille destinée à positionner de manière très générale les IHM sur des échelles de traitement naturel / technologique : tension allocutive (selon que l'on mobilise ou pas les formes d'adresse et de civilités des relations entre humains), tension interactionnelle (selon que l'interaction tende vers la requête unilatérale ou qu'elle se complexifie sous forme de conversation) et tension énonciative (selon que les formes langagières utilisées par l'un et l'autre des participants à l'échange tendent vers le code informatique ou des formes de dialogue humain).

\subsection{Google home : tension naturelle}

Le dispositif OK Google tend vers la sphère naturelle qu'il valorise. 
Ainsi, dans cet exemple extrait du site ${ }^{2}$ de la firme à fin promotionnelle :

\section{Ok Google, commence ma journée}

\section{Bonjour Chloé, ça fait plaisir de vous entendre !II est actuellement $15 \mathrm{~h}$, en ce moment à Paris il fait 18 degrés avec un ciel dégagé. Aujourd'hui vous avez prévu "Rendez-vous chez le médecin".}

57 Si le dispositif fonctionne en fait sur le mode de la requête/réponse à un tour (tension interactionnelle), les autres éléments de tension travaillent à la mobilisation des signes et indices de la conversation interpersonnelle. Ainsi, les partenaires s'appellent (« Google », "Chloé ») et se répondent de manière civile (" ça fait plaisir de vous entendre »), faisant tendre l'échange vers des formes d'allocution humaines; pendant que la tension énonciative vise à faire passer au second plan des énoncés techniques (tel le « Ok Google ", qui marque la sortie de veille du dispositif) au profit d'une énonciation ancrée (« aujourd'hui », « actuellement », « en ce moment ») supposant un partage d'expérience spatiale et temporelle.

Même limité, il s'agit bien d'un dialogue interpersonnel qui est mis en scène, incluant un partage d'expériences ontologiques (le temps, l'espace), de caractéristiques personnelles (nom, réseaux...), de savoirs partagés (un rendez-vous chez le médecin...).

Ce niveau de traitement dépasse donc largement celui de la gestion d'occurrences lexicales et sémantiques. Il ne s'agit plus de perfectionner des modes d'association d'éléments linguistiques (des processus anaphoriques par exemple) mais bien d'intégrer des éléments référentiels, personnels et expérientiels collectés et mis en bases de données, alors qu'en surface sont mobilisés, du fait du dispositif d'affordances, des simulacres de relations interhumaines.

Dans cette double interaction (informatique \& mécanique / interpersonnelle \& naturelle) établie en milieu ambiant (et non plus face à un appareil), se joue la mise en algorithme non seulement de la langue mais également du langage pour fonder un espace de convivialité dans ses dimensions sociales, relationnelles, sémiurgiques (Barthes ${ }^{3}$ ).

\subsection{Traits du dispositif conversationnel}

61 Songeant au développement de dispositifs d'interactions, il n'y a rien de surprenant à ce que les concepteurs s'inspirent des interactions existantes afin de permettre une fluidité dans les usages. Cependant, le caractère non humain de l'un des interlocuteurs pose la question de la mobilisation de ces ressources, à la fois dans la structuration conversationnelle (séquençage, histoire conversationnelle, multicodicité...) et dans le travail de la relation (personnalisation, rôles interactionnels, réseau relationnel) car elles convoquent de nouvelles postures et identités des sujets parlants.

62 Il ne s'agit pas de proposer ici une analyse exhaustive du dispositif mais de pointer, à travers certains traits saillants les emprunts de ces dispositifs d'interaction technologiques aux conversations humaines. 


\subsubsection{Structuration naturelle} réseaux d'amis, les habitudes... mais aussi la position géographique et ou les services et commerces préférés de l'utilisateur afin de lui offrir, selon l'expression commerciale, une « expérience personnalisée ».

Nous collectons des données destinées à rendre nos services plus rapides, plus intelligents, plus pertinents et plus utiles pour vous. Google Home s'améliore au fil du temps pour vous fournir des suggestions et des réponses plus adaptées et plus personnalisées [...] Google Home s'appuie sur les informations auxquelles vous lui avez donné accès pour améliorer la pertinence des réponses de l'Assistant Google [...] Nous enregistrons vos historiques de conversations pour adapter nos services à vos besoins et les rendre plus rapides et plus intelligents [...] etc.

(https://support.google.com/googlehome/answer/7072285, consulté le 30/12/2017).

La captation des données personnelles est donc une entreprise entièrement tournée vers le bénéfice consommateur qui, par ce partage historique (de navigation et de conversation via les différents services) est censé se trouver mieux protégé par Google 
Home contre les entreprises malveillantes : "Cela nous aide également à vous protéger des logiciels malveillants, de l'hameçonnage et d'autres activités suspectes » (sic).

La multicodicité des interactions humaines est également présente dans ces dispositifs. Elle prend la forme d'une déclinaison des codes sur les supports variés. Le cœur du dispositif Google Home est le programme «Assistant» (celui qui répond aux questions posées par l'utilisateur) qui se retrouve, outre dans l'appareillage domotique, de manière obligatoire sur les appareils utilisant le système d'exploitation Android. Ce système d'exploitation équipe près des trois-quarts des téléphones, phablettes et tablettes). Les utilisateurs du système d'application d'Apple ou de Microsoft trouveront l'équivalent (respectivement nommés Siri et Cortana). Nous sommes ici au cœur du principe pervasif, qui permet à l'utilisateur de s'affranchir de l'appareil pour retrouver son environnement partout, notamment grâce aux technologies du cloud computing (informatique dans les nuages) qui stockent à distance fichiers, applications, configurations de profils, données...

Ainsi, l'utilisateur n'est plus face à une machine mais au cœur d'un environnement connecté.

Il est alors possible de suivre un échange avec le dispositif sur plusieurs appareils, et surtout d'avoir des réponses variées du point de vue du code, puisque l'assistant Google peut répondre de manière vocale, par image ou texte (renvoi à des pages du web), ou même en effectuant une action (allumer la télévision connectée par exemple). Cela, en attendant les formes hologrammiques dont les fabricants nous assurent qu'elles ne devraient pas tarder à apparaitre dans les domiciles. De la même manière que les êtres humains peuvent se rencontrer dans la rue, se téléphoner, s'écrire... les interactions entre l'assistant Google et l'utilisateur peuvent prendre des formes variées et suivies en raison de la collecte des données qui constituent une histoire conversationnelle.

Une autre conséquence de cette pervasivité a trait à la mobilisation des ressources d'interaction complexes chez l'humain, dont le corps est également sollicité. L'utilisateur se tourne vers l'appareil Google Home pour lui adresser la parole, il se penche sur l'écran de son téléphone pour lire ses réponses...

Ces aspects qui pourraient, aujourd'hui, paraitre anecdotiques seront demain prépondérants, notamment en raison de la déclinaison des interfaces qui constitue un des défis technologiques majeurs actuels: généralisation des dispositifs tactiles, développement des interfaces olfactives... qui accentueront l'enveloppe numérique de l'existence humaine.

\subsubsection{Eléments de travail de la relation}

74 Au-delà de la forme et de la structure de la relation, ces dispositifs travaillent à établir une relation entre le produit et le consommateur en activant certains ressorts des relations interpersonnelles.

75 Un premier aspect a trait à la personnalisation des dispositifs qui combinent, rappelonsle, des appareils et des programmes. Inscrite (cf. 2.2.1. plus haut) au cœur de la naturalité, cette personnalisation prend des formes à la fois variées et récurrentes. L'affirmation publicitaire joue de ce traitement sémantiquement parfois jusqu'à l'extrême ;

Cette personnalisation est souvent articulée à un nom de marque présenté comme nom propre (Siri, Cortana, Alexa...), car, pour Serge Tisseron : 
L'introduction d'un nom incite à prêter à la machine une personnalité propre dans la mesure où celle-ci est alors identifiée exactement de la même manière qu'un individu est nommé dans la vie courante. Cette personnalisation met en valeur l'individualité d'une machine et renforce son incarnation en personnage (2015, p. 43).

77 Pour Google Home, le cas est différent. La marque Google a choisi de ne pas distinguer nominativement son produit. L'assistant est alors interpellé par l'utilisateur comme «Google ». Il faut dire que la firme peut se prévaloir d'un nom de marque qui fonctionne aussi bien comme nom propre que comme nom commun (le Petit Larousse 2014 accueille depuis 2014 Googler et Googliser, pour «signifier faire des recherches»), comme cartographe (Bouée, 2014, p. 21) hégémonique des connaissances et des accès. De même, elle ne joue pas sur l'attribution du genre qui anime par exemple l'assistant numérique de Microsoft (Perea, 2018).

78 Google se distingue ainsi de la concurrence en ne déployant pas un discours de personnification. Cependant, cette dernière n'est pas absente: le choix d'une voix humanisée qui est au cœur du dispositif Google Home est marqué par la captation des modes de communication interpersonnelle. De ce point de vue, on peut dire que ce qui distingue Google dans le positionnement de son produit, c'est le choix d'une personnalisation neutre et hégémonique.

79 Cette neutralité va peser sur les places et rôles interactionnels sous-tendus par le dispositif. Si d'autres jouent le rôle de l'altérité, à l'instar de la publicité pour le téléphone Mate 10 Pro (« doté d'une intelligence artificielle [...] il n'est pas artificiel, il est humain », comme annoncé dans une publicité télévisée de la marque Huawei, diffusée en décembre 2017 sur les chaines nationales françaises), cela n'est pas le créneau occupé par Google Home qui s'inscrit clairement dans des relations interlocutives (Vion, 1996) dissymétriques. En effet, l'initiative de la prise de parole est toujours du côté de l'utilisateur, les actes du langage «contraignent " l'appareil à répondre, et le mode conversationnel est de l'ordre des interactions de service.

80 A l'inverse, dans certains dispositifs ludiques, tels « Hello Barbie », la poupée connectée et parlante de Mattel, c'est la machine qui pose les questions pour ne pas avoir à répondre aux questions des utilisateurs et donner l'impression d'une conversation fluide.

81 Si aujourd'hui les dispositifs se présentent comme des " agents de renseignement » prêts à rendre un service informatif ou comme des dispositifs relationnels, selon la finalité commerciale du produit, nul doute que le développement de l'intelligence artificielle permettra très prochainement de proposer à ces agents conversationnels d'être totaux et de remplir des fonctions utilitaires et affectives à la fois. La présentation du dispositif Amazon Echo par le magazine «Objet connecté» est de ce point de vue assez enthousiaste :

Et si vous vivez seul, vous pouvez converser avec Alexa [nom de l'assistant-e virtuelle d'Amazon Echo]. Elle comprend tout ce que vous dites, tant que vous utilisez un langage simple et courant. Restez poli aussi, c'est quand même mieux. Les machines aussi ont droit au respect après tout. [...] Dites "Alexa" et de sa douce voix, elle vous dira tout ce que vous voulez savoir. [...] Elle fera intégralement partie de votre quotidien et pourra vite devenir aussi indispensable qu'un membre de votre famille et peut-être même plus utile ( https://www.objetconnecte.net/amazon-echo/, consulté le 1er janvier 2018). 
suffit pas qu'une relation interlocutive s'instaure entre des dispositifs dotés de personnalités, il faut encore qu'ils partagent des réseaux relationnels et des connaissances communes. Ces réseaux constituent une extension du domaine de la pervasivité : à la couverture totale dans l'espace et le temps (continuité de la connexion et de l'accès) se superpose la couverture totale des liens entre les personnes. Le dispositif devient ainsi un connaisseur du réseau capable d'apprendre pour associer un nom et des coordonnées complètes à « mon patron » ou " ma femme », de savoir grâce aux abonnements familiaux les appareils utilisés par les membres d'une famille...

Ces inferences anaphoriques et implicites relationnels font écho à ce que l'on retrouve dans les relations interhumaines. Ici, et même si le prétexte de la personnalisation est toujours mis en avant, cette cartographie du réseau relationnel est utile à fournir des bases de données monnayables par l'entreprise.

D'une manière générale, il n'y a pas à s'étonner que les dispositifs nouveaux empruntent à l'existant, et que les interactions vocales homme-machine s'inspirent des conversations humaines. Cependant, il faut noter que ces dispositifs se déploient toujours dans une double direction: celle de l'ergonomie d'usage mise en avant comme bénéfice consommateur et celle d'une collecte de données (liée à la croyance qu'une mise en algorithme des comportements et des pensées est possible) plus discrète car à fin de manipulation commerciale. Ce traitement est d'autant plus important qu'il n'est plus possible d'y échapper dans le temps et dans l'espace car notre environnement matériel et symbolique (espaces, langage, échanges...) est saturé de ces dispositifs pour une récupération et un traitement algorithmique de ces données conduisant à des décisions automatisées : que vais-je écouter comme musique, qui serait un(e) partenaire de vie idéal (e) ou comment réaliser des déplacements en ville?

85 Ces champs de décisions largement orientés hier comme aujourd'hui par les relations interpersonnelles connaissent ainsi une concurrence nouvelle : au conseil personnalisé se substitue l'incitation sur la base d'un comportement statistique. Cette concurrence est d'autant plus marquée qu'elle emprunte (de manière débutante aujourd'hui mais de plus en plus prégnante à l'avenir) à une activité humaine fondamentale et distinctive pour l'espèce jusqu'à ce jour: les interactions verbales. Une nouvelle étape de la grammatisation est franchie qui questionne l'identité humaine des sujets parlants.

\section{Conclusion}

Les interactions homme-machine en milieu pervasif s'inscrivent, malgré l'adjectif naturel qui leur est accolé, dans un rapport non transparent au langage humain, déterminé par des impératifs et des a priori technologiques. Elles s'inscrivent dans une histoire de technologie langagière qui conduit à la mécanisation comme ultime étape de la grammatisation, et repose sur une vision du langage réduit à ce qui est implémentable car, comme l'écrit Bruno Bachimont, en matière numérique "pour être traité, les événements du monde disparaissent derrière leur potentiel formatage » $(2014, \mathrm{p}$. 73). Dès lors, les options du langage sont réduites en raison des exigences de binarisation finale, stade profond nécessaire à son traitement automatique. Cette réduction permet d'envisager de nouveaux sujets parlants intelligents non humains dont la puissance de calcul permet néanmoins de simuler la complexité des interactions entre sujets humains.

Corela, HS-28 | 2019 
87 La question se pose alors du rapport entre ces sujets parlant, humains et non humains. Deux lignes d'observation ont été envisagées ici :

88 - la diffusion de ces rapports dans un environnement où l'intelligence est dite ambiante, où se couplage est permanent, où l'interlocuteur n'est plus un dispositif artefactuel (un ordinateur, un téléphone, un GPS...) mais consiste en une présence virtuelle permanente, identifiée, nommée, scénarisée ;

89 - la transaction entre mécanisation des conduites humaines (individu à l'identité configurée en données et adaptant ses productions langagières au dispositif) et naturalité des fonctionnements des appareils (personnification et simulacre des échanges interhumains).

Dans cette double tension, les sujets humains sont appelés à mobiliser, dans des processus d'affordance, les dispositifs ordinaires conversationnels qui constituent la base de la communication et de la médiation sémiotique. Les éléments de structuration qui les composent (séquençage, histoire conversationnelle, muticodicité) et le travail de la relation (personnification, les places et rôles interactionnels et la gestion des réseaux relationnels) sont dans l'histoire de l'espèce et de l'individu aussi ordinaires que largement tacites.

91 Ce travail de leur relation, pris dans l'entrelacement de la nature et l'artifice du langage, conduit alors à questionner la nature et l'artifice de la nature humaine. Si le langage doublement articulé a fait la spécificité de l'humanité, que reste-t-il de cette identité quand cette distinction n'est plus?

92 Il n'est peut-être pas anodin qu'au-delà de la caricature d'ambitions prothétiques, des humanoïdes de science-fiction et les développements des robots de compagnie... la question du post-humanisme se pose avec urgence. Ce dispositif n'oppose plus vivant et non vivant, être humain et machine... mais les saisit dans les bornes d'un continuum, dans une transaction identitaire permanente.

93 Si le Test de Turing évaluait la capacité de la machine à leurrer le locuteur humain incapable de déterminer s'il converse avec un semblable ou avec un programme, les travaux actuels sur la naturalité des échanges semblent montrer que, plus qu'un " simple » mimétisme trompeur de la machine, c'est un travail de co-détermination qui est à l'œuvre. En même temps que la machine se met à parler comme l'homme, celui-ci doit se mettre à parler comme à une machine s'il veut se faire entendre. Cela est d'autant plus vrai que l'écoute sera de plus en plus permanente et continue, dans les pratiques privées comme dans les pratiques professionnelles, à la maison comme au travail, à plusieurs ou en tête-à-tête, etc. Pour faciliter le dialogue, les industriels travaillent au naturalisme, en même temps que les êtres humains révèlent leur part technologique qui transparaît dans ces échanges.

\section{BIBLIOGRAPHIE}

AUROUX Sylvain, 1996, La philosophie du langage, Paris, PUF. 
AUROUX Sylvain, 1994, La révolution technologique de la grammatisation, Liège, Mardaga.

BACHIMONT Bruno, 2014, « Le nominalisme et la culture : questions posées par les enjeux du numérique ", dans Digital Studies. Organologie des savoirs et technologies de la connaissance, sous la direction de Bernard Stiegler, éd. Fyp, p. 63-78.

BOUÉE Charles-Edouard, Confucius et les automates. L'avenir de l'homme dans la civilisation des machines, Paris, Grasset.

CHASTAGNOL Clément, CLAVEL Céline, COURGEON Matthieu et DEVILLIERS Laurence, 2014, « Designing an Emotion Detection System for Socially Intelligent Human-Robot Interaction », in J. Mariani, S. Rosst, M. Garnier-Rizet et L. Devilliers (Eds.), Natural Interaction with Robots, Knowbots and Smartphones, New-York, Springer, 199-212.

COOREN François, 2010, « Ventriloquie, performativité et communication. Ou comment fait-on parler les choses », Réseaux 2010/5, n 163, p. 33-54.

COUTAZ Joëlle, CROWLEY James L., 2008, « Plan « intelligence ambiante » : défis et opportunités ", en ligne : http://iihm.imag.fr/publs/2008/RapportIntellAmbiante.V1.2finale.pdf, consulté le 5/8/2017.

DEVELOTTE Christine, KERN Richard et LAMY Marie-Noëlle, 2011, Décrire la conversation en ligne. Le face à face distanciel, ENS Editions.

DUNBAR Robin, 1998, Grooming, Gossip and the Evolution of Language, Cambridge, Harvard University Press.

GOLOPENTIA-ERESCU Sandra, 1988, « Interaction et histoire conversationnelle », Échanges sur la conversation, J. Cosnier, N. Gelas et C. Kerbrat-Orecchioni (Dir.), Paris, éd. CNRS, p. 69-81.

GUCHET Xavier, 2008, «Évolution technique et objectivité technique chez Leroi-Gourhan et Simondon ", Appareil [En ligne], n², mis en ligne le 11 septembre 2008, consulté le 08 août 2017. URL : http://appareil.revues.org/580

DEBRE, Régis (2000), «Les révolutions médiologiques dans l'Histoire. Pour une approche comparative », Bulletin des Bibliothèques de France, 1(45), p. 4-12.

DE FORNEL Michel, LEON Jacqueline. « L'analyse de conversation, de l'ethnomethodologie à la linguistique interactionnelle », dans Histoire Épistémologie Langage, tome 22, fascicule 1, p. 131-155 GIBSON James Jérôme, 1977, The Theory of Affordances. In Perceiving, Acting, and Knowing, Eds. Robert Shaw and John Bransford, (ISBN 978-0-470-99014-8)

GIBSON James Jérôme, 1986, The Ecological Approach to visual perception. Hillsdale, NJ : Lawrence Erlbaum Associates, Inc. (Original work published in 1979).

HALADJIAN Rafi, 2003, De l'inéluctabilité du Réseau Pervasif, Ozone.

HAYLES Katherine, 2016, Lire et penser en milieux numériques. Attention, récits, technogenèse, Grenoble, éd. Ellug. (Edition originale : 2012, How we think : Digital Media and Contemporary technogenesis, Chicago, Chicago University Press).

HARRISON Deborah, 2016, conférence donnée le 28 janvier 2016 au Re-Work - Virtual Assistant Summit de San Francisco, en ligne : https://www.youtube.com/watch ?v =-WcC9PNMuL0, consultée le 20/07/2016.

HUTCHBY I., 2001, Conversation and Technology : from the Telephone to the Internet, Cambridge, Polity Press. 
JOUËT Josiane, 2000,, " Retour critique sur la sociologie des usages », Réseaux, n 100, vol. 18, 487-521.

LATOUR B., 1997, Nous n'avons jamais été modernes. Essai d'anthropologie symétrique, Paris, La Découverte.

LEON Jacqueline, 2015, Histoire de l'automatisation des sciences du langage, Paris, ENS éditions.

LEON Jean, LUZZATI Daniel et POIBEAU Thierry (dir.), 2014, « Traitements automatiques de l'oral et de l'écrit. Quelle place pour la linguistique? ", L'information grammaticale, $\mathrm{n}^{\circ} 142$.

LUZZATI Daniel, ADDA-DECKER Martine et ROSSET Sophie (dir.), 2014, « Traitements automatiques de l'oral et de l'écrit. Panorama des recherches et des technologies actuelles ", L'information grammaticale, $\mathrm{n}^{\circ} 141$.

MALDIDIER Denis, (présentatrice), 1990, L'inquiétude du discours. Textes de M. Pêcheux, Éd. des Cendres, $336 \mathrm{p}$.

NORMAN Donald, 1989, The Design of Everyday Things, Doubleday Currency

PAVEAU Marie-Anne, 2012, «Ce que disent les objets. Sens, affordance, cognition », Synergie, Parys riverains de la Baltique, $n^{\circ}$ 9, p. 53-65.

PEREA François, 2016, « Nature et technologie langagière dans les dialogues oraux hommemachine ", Communication [En ligne], vol. 34/1, mis en ligne le 26 août 2016, consulté le 05 août 2017. URL : http://communication.revues.org/6763 ; DOI : 10.4000/communication.6763

PEREA François et RICHARD Arnaud, 2018, « De qu(o)i Siri est-il le nom ? », Le discours et la langue, t. 10.1 .

PEREA François, 2018, « Cortana est-elle une femme comme les autres ?», Semen, n 44, p. 61-79. SADIN Eric, 2013, L'humanité augmentée. L'administration numérique du monde, Paris, L'Echappée belle, Coll. « Pour en finir avec».

STIEGLER Bernard, 1998, " Leroi-Gourhan : l'inorganique organisé », Cahier de médiologie, n 6, p. 187-194.

STIEGLER Bernard, 2013, " Autonomie et automatisation dans l'épistémè numérique », communication à l'Agence nationale de la recherche, en ligne : http://www.agence-nationalerecherche.fr/Colloques/RencontresduNumerique/fichiers/Steigler.pdf, consulté le 14/08/2017.

TISSERON S., 2015, Le jour où mon robot m'aimera. Vers l'empathie artificielle, Paris, Albien Michel.

TOURAINE Damien, 2003, Interaction "naturelle » en environnements immersifs - Démonstrateur multimodal et validation sur des applications scientifiques. Interface homme-machine, Thèse en sciences de l'Université Paris Sud - Paris XI.

TURING Alan, 1950, «Computing machinery and intelligence », Mind, Oxford University Press, 49 : 433-460.

VION Robert, 1996, «L'analyse des interactions verbales », Les Carnets du Cediscor , n 4.

VICTTORI Bernard, 1996, « Les systèmes informatiques doivent-ils modéliser leurs utilisateurs ? ", in J. Vivier (ed.), Psychologie du dialogue homme-machine en langage naturel, Paris, Europia.

WEISER Mark, 1991, « The Computer for the Twenty-First Century », Scientific American, vol. 265, $\mathrm{n}^{\circ} 3$, p. 94

ZELKHA Eli, EPSTEIN Brian, BIRRELL Simon et DODSWORTH Clark, 1998, "From Devices to "Ambient Intelligence", Digital Living Room Conference (published June 1998) 


\section{NOTES}

1. Au sens écologique du terme désignant ici l'adaptation des technologies au monde préexistant, mais également leurs incidences en retour de cette intégration.

2. https://store.google.com/product/google_home_learn, consulté le 18/12/2017.

3. Nous reprenons le terme « sémiurgie » forgé par Roland Barthes (1976, p. 180), qui désigne non pas l'étude des signes (sémiologie), mais qui met l'accent sur l'action, la création démiurgique de sens, la fabrication et l'utilisation de signes en vue de produire certains effets. BARTHES R., $S$ / $Z$, Paris, Seuil, Points, 1976.

\section{RÉSUMÉS}

L'évolution industrielle nous destine à vivre dans des environnements technologiques caractérisés par une dissémination informatique totale dans la multitude et d'objets connectés capables de traitement sémantique et d'interaction informationnelle avec les humains. Dans ce contexte d'Intelligence ambiante (Zelkha et al., 1998) se déploient des dispositifs domotiques d'Interactions dites naturelles Homme / machine. Ainsi de Google Home (dont la présentation en ligne par la firme sert de corpus pour le présent travail) présenté comme : " une enceinte à commande vocale qui fonctionne avec l'Assistant Google » (https://madeby.google.com/home/, consulté le 04/10/2017). On pourra tout aussi bien discuter avec les agents de la concurrence : Amazon Echo, Apple HomePod, etc. Ces dispositifs nous conduisent à interroger une étape contemporaine majeure de " la révolution technologique du langage " (Auroux, 1994) et de son automatisation (Léon, 2015), qui fait des machines de réels sujets parlants, inscrits dans un " couplage humano-machinique "(Sadin, 2013). Dans ce contexte fortement marqué par les affordances (Gibson, 1977), nous observons les modalités de cette interaction artéfactuelle et dite naturelle, caractérisée notamment par une simulation conversationnelle et une personnalisation des dispositifs technologiques. La perspective est celle d'une linguistique post-dualiste et non logocentrée, dans la lignée du courant de l'analyse du discours (en interaction) : Texte-DiscoursInteraction (Paveau, 2012).

Nowadays, our technological environment is characterized by a total computer dissemination in the intelligent and connected objects. In this context of Ambient Intelligence (Zelkha et al., 1998) the domotic devices are deployed in so-called " natural" interactions with humans. For example Google Home is presented as: "a voice-activated speaker that works with the Google Assistant" ( https://madeby.google.com/home /, accessed 04/10/2017). We can also discuss with the competition agents: Amazon Echo, Apple HomePod, etc. These devices lead us to question a new step of "the technological revolution of language" (Auroux, 1994) and its automation (Léon, 2015), which makes machines real talking subjects, enrolled in a "human-machine coupling" "(Sadin, 2013). In this context of affordances (Gibson, 1977), we observe the modalities of the H/ $\mathrm{M}$ interaction, characterized in particular by a conversational simulation and a personalization of the technological devices. Our perspective is post-dualistic and non-logocentric, in line with the current of discourse analysis (in interaction): Text-Speech-Interaction (Paveau, 2012). 
INDEX

Mots-clés : Interaction naturelle homme / machine, affordance, conversation, technologies langagières

Keywords : Natural interaction uman / machine, affordance, conversation, language technologies

\section{AUTEUR}

FRANÇOIS PEREA

Université Paul-Valéry Montpellier 3, Praxiling UMR 5267 